current level of understanding of these properties, calling for the most part on models developed to describe amorphous semiconductors. For liquids, of course, further difficulties arise. The local atomic configuration and hence the electronic structure changes not only with time but also with temperature. Thus, if measurements are made as a function of temperature, one has to deal with a different material at each temperature.

The field is not short of experimental results and these are presented in several chapters, one devoted to physical properties, one to physicochemical properties and metallurgical properties, and two others to specific alloys. The book is valuable for this collection alone. It is not surprising to find T1-Te alloys discussed extensively, since this system has not only been exhaustively investigated by the author himself, but also exhibits so many of the diverse and interesting properties characteristic of liquid semiconductors. Expcrimentalists will appreciate the short chapter on techniques.

\section{Group theory or general relativity}

Group Theory and General Relativity. By Moshe Carmeli. Pp. 391. (McGrawHill: New York and Toronto, 1977.) $£ 16.85$.

THERE is no doubt that a textbook which links together the topics of group theory and general relativity is very welcome. The jacket of this book proclaims that it is the first book on "the applications of group theory to general relativity", but I have some reservations about whether this is an appropriate description. Reading the book gives the impression of two research monographs grafted together: one on group theory, the other general relativity. This does not mean that the contents are not useful, but some stronger motivation for the conjunction of topics would have been desirable.

Field theoreticians are well used to relying heavily on group theoretical techniques, particularly with the recent activity in the area of gauge theories. When it comes to general relativity, group techniques are less powerful because of the absence in general of metric-preserving global diffeomorphisms. Of course, interest centres on special model spacetimes with particular symmetries, and group theory has long played an elegant and useful role in elucidating and classifying their properties. More general classes of
It behoves a reviewer to draw attention to faults lest it be thought that he is a friend of the author (which he is) or else in league with the publishers (which he is not). My only criticism (apart from an unfortunate interchange of the words band and bond between two section headings in the list of contents), is that tantalising extracts of theory and models, described in detail in later chapters, are interspersed with experimental data presented earlier in the book; yet if the reader tries the theoretical chapters first, he is confronted with tantalising extracts of data. One has to learn, however, to find one's way around any book of this kind and specialists in the field will certainly have no trouble.

The price came as a pleasant surprise (by today's standards) and the book has to be good value for such an admirable condensation of a difficult subject by a leader in the field.

E. A. Davis

E. A. Davis is Lecturer in Physics at the University of Cambridge, UK.

spacetimes, however, can be fruitfully investigated using group methods. Especially important are those that admit asymptotic symmetry groups, and the formulation of concepts such as asymptotic energy momentum, and multipole moments of the gravitational field for isolated bodies relies heavily on the analysis of these groups. In addition to this, much attention has recently been directed to gauge theories in curved spacetime, in which the gauge group continues to play an important part.

This book covers much of the material relevant to these applications, but in a somewhat shapeless form. Readers who have no previous knowledge of one or the other (or both) central topics may find the presentation a little stark with essential facts simply run off. I would recommend some previous background reading, at least for the group theory, from the books of Naimark or Gel'fand et al. These are recommended by the author in his introduction, and Carmeli's treatment is closely similar to these.

The first six chapters contain little general relativity, dealing extensively with the representations of the rotation and Lorentz groups. Chapter 3 treats the spinor representations of the Lorentz group. Chapters 4, 5 and 6 tackle in great depth the principal, complementary and complete series of representations of $\mathrm{SL}(2, \mathrm{C})$.

The author's discussion of relativity begins with chapter 7 . The treatment is standard and traditional, and no attempt seems to have been made to present the material in any especially novel or enlightening way, or to exploit many of the techniques of modern differential geometry. It is here that one most feels the need for stronger links with the group theory and motivating remarks. The author goes on to develop a detailed discussion of the spinor formulation of general relativity, and it is particularly useful that this approach, advocated by Roger Penrose, and for so long in widespread use among relativists, is dealt with in an integrated fashion in a student textbook. It is frustrating, though, that many useful results of spinor calculus are relegated to the exercises.

In chapter 9, Carmeli takes up the subject of gauge theories, and after a preliminary discussion of electromagnetic and Yang-Mills fields attempts to formulate general relativity also as a gauge theory. The Newman-Penrose formalism is obtained from a gauge theory approach, though perhaps its physical relevance could have been better described. The Goldberg-Sachs theorem is proved in chapter 10 , but its importance is not made transparent.

The final two chapters of the book deal with solutions of the field equations of general relativity and the Bondi-Metzner-Sachs group. There are also several appendices. Once again, all the details are competently collected together, but the unitiated reader may have difficulty in understanding their wider importance or relevance. Perhaps these people would do well to consult the final paragraph of the Introduction which contains a quotation of Abdus Salam, who learned group theory from Racah: "After attending these lectures I thought this is really too hard; I cannot learn this; one is hardly ever likely to need all this complicated matter. I was completely wrong".

In spite of the specific criticisms concerning the presentation, 1 find this book a timely addition to the many rescarch papers on these two most important topics. It should prove useful as a teaching text and, at least in conjunction with some background matcrial, it could be used as a course book for either group theory for physicists, or more specifically for relativity courses. The style is straightforward and information retrieval easy. Many exercises are given with each chapter and an extensive bibliography is added. It is elegantly produced, and at $£ 16.85$ is not over-expensive for a mathematical book of this length.

Paul Davies

Paul Davies is Lecturer in Mathematics at King's College, University of London, $U K$. 